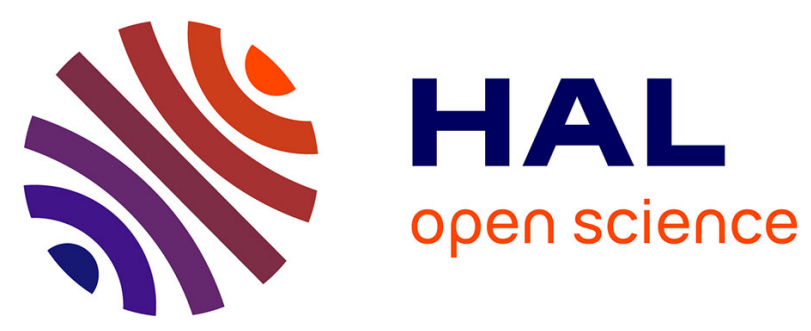

\title{
Constructing the topology of complex wire networks using reflectometry response and Newton-based optimization algorithms
}

\author{
Moussa Kafal, Jaume Benoit, Geoffrey Beck, Antoine Dupret
}

\section{To cite this version:}

Moussa Kafal, Jaume Benoit, Geoffrey Beck, Antoine Dupret. Constructing the topology of complex wire networks using reflectometry response and Newton-based optimization algorithms. 2017 International Automatic Testing Conference (AUTOTESTCON), Sep 2017, Schaumburg, United States. pp.8080495, 10.1109/AUTEST.2017.8080495 . cea-01837006

\section{HAL Id: cea-01837006 https://hal-cea.archives-ouvertes.fr/cea-01837006}

Submitted on 9 Dec 2020

HAL is a multi-disciplinary open access archive for the deposit and dissemination of scientific research documents, whether they are published or not. The documents may come from teaching and research institutions in France or abroad, or from public or private research centers.
L'archive ouverte pluridisciplinaire HAL, est destinée au dépôt et à la diffusion de documents scientifiques de niveau recherche, publiés ou non, émanant des établissements d'enseignement et de recherche français ou étrangers, des laboratoires publics ou privés. 


\title{
Constructing the Topology of Complex Wire Networks Using Reflectometry Response and Newton-Based Optimization Algorithms
}

\author{
Moussa Kafal, Jaume Benoit, Geoffrey Beck, Antoine Dupret \\ CEA, LIST, Laboratoire de Fiabilisation et d'Intégration des Capteurs \\ Nano-Innov, Bât. 862-PC172, 91191 Gif-sur-Yvette Cedex, France
}

\begin{abstract}
Based on Time-Domain Reflectometry (TDR) technique, a new method which could reconstruct the topology of an unknown or partially unknown network is proposed in this paper. This approach, the TDR-NEW approach, allows the network to be retrieved by drawing its interconnections and estimating the lengths of branches. Two complementary steps are addressed. In the first step the direct problem is modeled using RLCG circuit parameters. Then the Finite Difference Time Domain (FDTD) method conjugated with the ABCD matrix method is used to model the signal propagation in a branched network. This model, the FDTD-ABCD, provides a simple and accurate method to simulate Time Domain Reflectometry responses. In the second step the newton-based optimization method is combined with the wire propagation model to solve the inverse problem and to deduce physical informations about the network from the reflectometry response. Numerical and experimental results pointing out the performance of the TDR-NEW method in reconstructing the topology of branched networks are reported.
\end{abstract}

Keywords-Wiring networks, topology reconstruction, time domain reflectometry, newton-based optimization algorithms.

\section{INTRODUCTION}

Fundamental changes are taking place in the monitoring, control and protection of almost all modern systems where an increasing amount of electrical cables are progressively and massively hosted [1]. On the other hand, it is also generally acknowledged that electrical systems are evolving and becoming more complex accompanied by the enormous increase of electrical employments, which require new transmission and distribution lines [2]. Due to their ubiquitous use in any small, medium or large infrastructure (e.g., smart grids, buildings, transport systems, vehicles, and power plants) and their primary role in energy and signal distribution, wire networks became fundamental subsystems whose proper functioning and good performance is of critical importance.

It is noteworthy that a great number of electrical wires are dedicated to safety and control operations, thus any shortening in their performance due to the appearance of a defect might be highly expensive in terms of lives and from an economical aspect. One estimate is that highly trained technicians spend between 1 million to 2 million man-hours each year at the operational level to troubleshoot and repair wiring system problems in the U.S navy alone [3]. Accordingly, ensuring the reliable use of wire networks requires the availability of techniques capable of detecting the presence of faults that could potentially put in jeopardy the infrastructures relying on these networks.

Fault location problems have been extensively studied in the literature for many years and numerous methods have been proposed for this purpose. Nevertheless, despite the vast amount of literature, the problem of fault location still represents a serious challenge. Although several electric and non-electric wire diagnosis methods have been studied and developed throughout the last few decades, reflectometry-based techniques are still in the center stage of research and industrial applications in this domain [4]-[7].

Reflectometry are high frequency methods adopted from the concept of radar that allow relatively simple test setups for the analysis of a network under test (NUT). Typically, a test signal is injected into the NUT which propagates until it encounters an impedance discontinuity, e.g., a branch junction, a load, or a local unwanted modification of the line, i.e., a fault. Hard faults (open or short circuits) prevent the test signal from ongoing throughout the NUT while soft faults e.g., a partial wearing of the insulation or a chafing, partially reflect it back to the injection point. The analysis of the reflectometry response provides valuable information about the fault (presence, position, and nature).

In general, the accuracy of reflectometry methods are mainly affected by several factors including: pre-fault system condition, network's topology, fault impedance, and presence of noise. In particular, the problem of soft faults is more critical and requires in-depth studies as their corresponding reflected weak echoes might be easily masked from reflectometry detection by pseudo echoes generated by higher reflectivity impedance discontinuities like junctions [8]. Although these methods have promising capabilities to locate faults on single cables, their applicability is declined on branched NUTs. In practice, cables are always used in distribution networks where a set of cables, usually of the same type, interconnect according to well defined topology standards (bus, star, mesh, ring, tree, etc.). As a matter of fact, testing such kinds of networks has proven to be challenging. This is because branches in a network add a further level of complexity to the reflectometry response due to the superposition of multiple reflections from different branches and branch ends [9]. The number of reflections also tend to grow exponentially with the number of branches in the network; hence, complex network topologies produce extremely complex reflectometry responses leading to ambiguous results.

Under those circumstances, developing new techniques that can be integrated with reflectometry methods to improve and enhance the analysis of the reflectometry responses became essential. Significantly, inverse problems showed to be suitable for this purpose, where baselining an approach relying on comparing the response of the faulty network with either the pre-measured or simulated response of its (known) healthy 
condition improved the performance of reflectometry [10]. The rationale for this method is based on the following observation: while echoes from soft faults are often dwarfed by those generated by, e.g., junctions, a baseline measurement contains exactly the same echoes from the same junctions; taking the difference of the two results is intended to cancel out the contribution from the NUT itself (i.e., the junctions in this example), making the fault response detectable. Baselining is indeed widely used in reflectometry as a contrast-enhancing technique, especially with complex branched NUTs and weak reflectivity soft faults [11]. Additionally, it formed an important basis for the application of the new trend of time reversal techniques that have shown to be efficient in locating soft faults in different complexity NUTS [12]-[15].

Consequently, it can be claimed that analyzing networks of unknown topology can therefore be particularly very difficult. In fact, wire networks have been deployed in many applications decades ago, as a result many of their datasheets might have been lost with time. Furthermore, there exists a wide range of networks that can be hardly accessed especially those that are either embedded or present in critical situations as nuclear power plants. Accordingly, testing such networks can sometimes be impossible.

In this paper we propose an efficient method for the reconstruction of wiring networks using the reflectometry response, particularly the time domain reflectometry (TDR), and newtonbased optimization algorithms. We are going to introduce and develop a technique for constructing an unknown network topology where the studied network will be considered as a black box having a definite number of ports. A direct model describes the propagation of the wave along the lines of a network using a numerical method. Then a newton-based algorithm is used to solve the inverse problem reducing the differences between the measured reflectometry response of the unknown NUT and the response given by the direct model. The result will be revealing the most compelling network topology while constructing all branches and junctions with the right cable lengths.

\section{The Wire Network NumericAl Model}

To better understand the tenets of the proposed approach in reconstructing the topology of an unknown network, it is necessary to understand how an electromagnetic wave propagates in a transmission line. The aim of this section is first to position our work within the framework of the transmission line theory and in the context of wiring modeling techniques and then present a method for modeling a wire network. For our case study we considered two-conductor transmission lines under the assumption that the electric and magnetic fields are transverse to the axis of propagation (TEM wave).

\section{A. The Transmission Line propagation Model}

In literature, the methods used to simulate and study the transmission line behavior are different. Most of them are obtained from the time dependent telegraphers equations (1) \&(2) modeled by RLCG circuit parameters for an infinitesimal length of the line. Notably, these equations describe the evolution of voltage and current along the transmission line as functions of the per unit length parameters of the line demonstrated by the quantities $R$ (resistance), $L$ (inductance), $C$ (capacitance) and $G$ (conductance).

In our work, we opted to use the finite difference time domain (FDTD) method in order solve telegraphers equations and model the signal propagation in a transmission line. The FDTD method is a simple and intuitive electromagnetic modeling technique which converts the differential equations into finite difference equations leading to the computation of the voltage and current propagation along a line.

$$
\begin{aligned}
& \frac{\partial v(x, t)}{\partial x}=-R i(x, t)-L \frac{\partial i(x, t)}{\partial t} \\
& \frac{\partial i(x, t)}{\partial x}=-G v(x, t)-C \frac{\partial v(x, t)}{\partial t}
\end{aligned}
$$

\section{B. Branched network analysis}

In real-life environments such as aerospace and automotive, wired networking interconnect dozens or even hundreds of electrical systems together. These wired networks consist of a set of wires, usually of the same type, interconnected according to well defined topology standards. Numerous transmission line techniques have been used for modeling wired networks, where we adopted a joint FDTD-ABCD method which formed a suitable solution for such analysis of branched NUTs. It is worthy to note that this model has been developed at CEA as an inhouse solver offering excellent fidelity and computational efficiency.

To model networks, transmission conditions have to be implemented at the junctions. Accordingly, for a uniform transmission line of characteristic impedance $Z_{c}$ ending by a load impedance $Z_{l}$, the reflection coefficient $\Gamma_{i}$ at any discontinuity $Z_{i}$ can be defined as the ratio between the reflected $V_{\text {reflected }}$ and incident $V_{\text {incident }}$ voltages as given in eq. 3. Certainly, hard faults whether open or short circuits result in an absolute reflection coefficient equal to $1\left(\left|\Gamma_{i}\right|=1\right)$, whereas any other kind of defect will result in a $\left|\Gamma_{i}\right|<1$. For instance, a junction branching into three wires having the same $Z_{c}$ as that shown in Fig. 1 results in a $Z_{i}=Z_{c} / 3$. It is important to note here that we are going to deal with networks composed of branches having the same characteristic impedance $Z_{c}$. In effect, the corresponding reflection coefficient of this junction will be $\Gamma_{i}=-1 / 2$. In general, for a junction of $n$ branches $Z_{i}=Z_{c} / n$, the reflection coefficient can be defined as shown in eq. 4 .

$$
\begin{gathered}
\Gamma_{f}=\frac{V_{\text {reflected }}}{V_{\text {incident }}}=\frac{Z_{i}-Z_{c}}{Z_{i}+Z c} \\
\Gamma_{i}=\frac{Z_{c}-\frac{Z_{c}}{n}}{Z_{c}+\frac{Z_{c}}{n}}=\frac{1-n}{1+n}
\end{gathered}
$$

\section{Reflectometry for a branched network topology}

In order to validate the propagation model in complex branched networks, we will consider the FDTD-ABCD model to simulate the time domain reflectometry (TDR) response of the four junction NUT of Fig. 1. The network is composed from 10 ideal non-dispersive branches with a characteristic impedance $Z_{c}=50 \Omega$ interconnected by four junctions. 
One of the network's extremities will then be selected to serve as a reference testing port and will be denoted as $P_{\text {test }}$. A normalized amplitude Gaussian pulse with a total bandwidth of $2 \mathrm{GHz}$ will be injected into $P_{\text {test }}$ of the NUT. Notably, all NUT extremities are matched to the characteristic impedance of the network. The corresponding TDR response is shown in the reflectogram of Fig. 2. The same pulse will be re-injected into the same network but this time while having open circuited extremities. The corresponding reflectogram is also presented in Fig. 2.

Several points need to be commented here. First of all, it shall be noted that for the case of matched end networks, the first two reflections of the TDR response are strictly attached to those of the first two consecutive junctions. Therefore, the first peak value is the first junction's reflection coefficient while its position corresponds to the distance at which it is located. On the other hand, the second peak value hints at the second junction's reflection coefficient according to the relation given by equation 5 where $\Gamma_{2}$ and $\Gamma_{2}^{\prime}$ are the actual junction's reflection coefficient and its peak value respectively. Accordingly, once $\Gamma_{2}$ is computed, the number of branches $n_{2}$ originating from it can be inferred from eq. 4 while its distance from $P_{\text {test }}$ is the location of its peak value.

$$
\Gamma_{2}=\frac{\Gamma_{2}^{\prime}}{\left(1+\Gamma_{1}\right)^{2}}
$$

As an illustration, if we reconsider the reflectogram of Fig. $2 \Gamma_{1}=-1 / 2$. Thus according to eq. $4 n_{1}=3$ while $l_{1}=$ $1.5 \mathrm{~m}$ is the first junction location from $P_{\text {test }}$. On the other hand, the second junction seems to be located at $l_{2}=4 \mathrm{~m}$ from the reference testing ports and interconnecting $n_{2}=2$ branches.

With this in mind, if we consider an unknown network embedded in a black box while having access to all its extremities, the TDR response computed from a single testing port while matching all other ends permits building a preliminary network configuration. In fact, we can infer according to what has been detailed before the location of the first two junctions along with the number of branches connected to each.

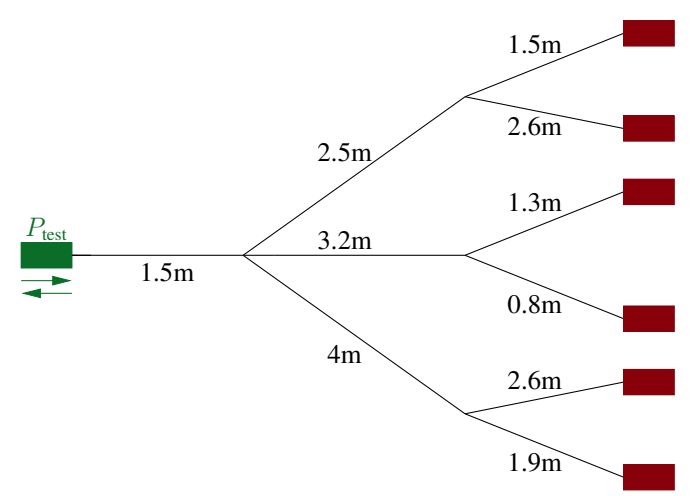

Fig. 1. Layout of the four Y-junction NUT considered for the numerical validation of the TDR-NEW approach including the branch lengths.

Due to the characteristics of wiring networks, the presence of multiple junctions and different load terminations provides multiple and intermediate reflections in the reflectometry response as shown in Fig. 2. These reflections occur because

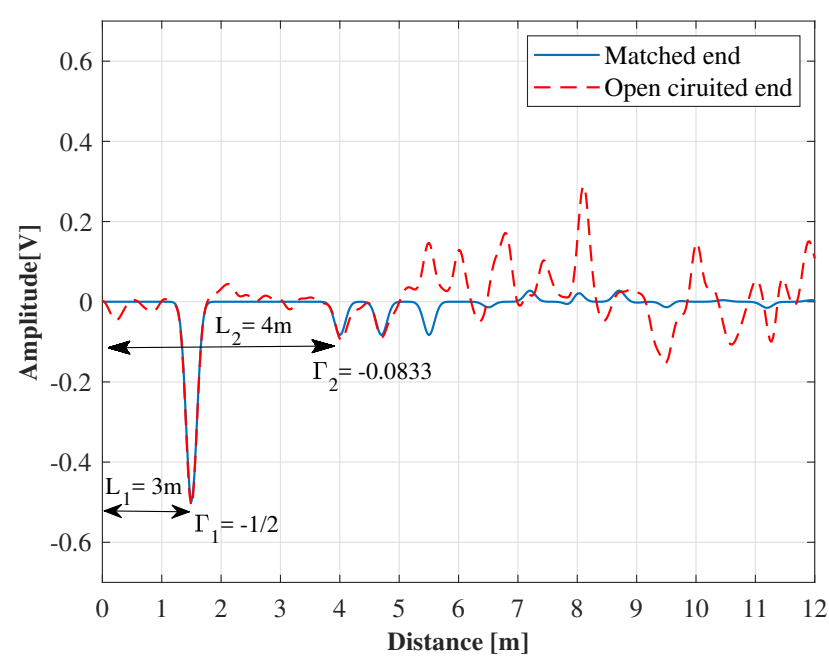

Fig. 2. The TDR reflectometry responses corresponding to the network of Fig. 1 in the cases of matched and open-ended extremities.

the primary reflections from the junctions and terminations intercept other junctions during their return path to the source. In these conditions, if the network topology is not known in advance, it is very difficult to deduce the length of the different branches from the reflectometry response. Thus the reflectometry response of an open circuited network alone is not sufficient to reconstruct a wiring network. The coming section introduces a novel method for the reconstruction of an unknown wiring network from reflectometry data based on the conclusions built from the matched end observations.

\section{THE PROPOSED TDR-NEW ALGORITHM}

The TDR-NEW approach consists of two main parts. The first part comprises a numerical model for constructing possible NUTs based on the matched end TDR methodology illustrated in section II-C. The second part is the newtonbased optimization which is used to minimize the differences between the measured open-ended reflectometry response of the unknown NUT and the responses given by the direct numerical model. The network's topology and its corresponding branch lengths would be retrieved once convergence is reached between the measured response and the one corresponding to the right NUT configuration generated by the numerical model.

\section{A. Newton's Method}

Newton's method is a basic tool in numerical analysis and numerous applications, including operations in data mining, management science, industrial and financial research, optimization and control. Its role in optimization cannot be underestimated as it is the basis for the most effective procedures in linear and nonlinear programming. For instance, the polynomial time interior point algorithms in convex optimization are based on Newton's method.

The basic idea of Newtons method is very simple: it is linearization. Suppose $F: R^{1} \rightarrow R^{1}$ is a differentiable function, and we are solving the equation $F(x)=0$. Starting from an initial point $x_{0}$ we can construct the linear 
approximation of $F(x)$ in the neighborhood of $x_{0}: F\left(x_{0}+\right.$ $h) \approx F\left(x_{0}\right)+F^{\prime}\left(x_{0}\right) h$ and solve the arising linear equation $F\left(x_{0}\right)+F^{\prime}\left(x_{0}\right) h=0$. Thus, we obtain by the recurrent method

$$
x_{k+1}=x_{k}-F^{\prime}\left(x_{k}\right)^{-1} F\left(x_{k}\right), \quad k=0,1,2 \ldots
$$

In optimization, Newton's method is applied to the derivative $F^{\prime}$ of a twice-differentiable function $F$ to find the roots of the derivative (solutions to $F^{\prime}(x)=0$ ), also known as the stationary points of $F$ [16].

\section{B. The TDR-NEW approach}

In order to illustrate the steps followed by the TDRNEW algorithm in reconstructing the topology of an unknown network, we are going to consider a random NUT built inside a black-box. We assume having no information about any of its connections except the knowledge of the number of extremities $M$. The following step is to assign a reference testing port $P_{\text {test }}$ where two TDR measurements will be done. The first will be accomplished while matching all the NUT's extremities whose corresponding reflectometry response will be denoted as $V_{\text {match }}^{\text {mes }}$. On the other hand, $V_{\text {open }}^{\text {mes }}$ will be the TDR response resulting from an open-ended network extremities measurement.

After having measured the matched end reflectometry response $V_{\text {match }}^{\text {mes }}$, the first part of the TDR-NEW approach begins by numerically constructing all $N$ possible NUT configurations $N U T_{\text {sim }}^{i}(i=1,2, \ldots, N)$ based on the knowledge of the NUT's $M$ extremities. In fact, $V_{\text {match }}^{\text {mes }}$ permits extracting the position and the number of branches of the first two junctions in the network $\left(l_{1}, n_{1}, l_{2}\right.$, and $\left.n_{2}\right)$ as previously detailed in II-C. Therefore, the number of possible NUTs would decrease as the number of free extremities $M$ diminishes. The TDR response $V_{\text {sim }}^{i}$ of each $N U T_{\text {sim }}^{i}$ is determined by the numerical model described section II.

The problem now is to select the $N U T_{\text {sim }}^{i}$ that best resembles the unknown studied network. Notably, $V_{\text {open }}^{m e s}$ allows retrieving different branch interconnections due to the multiple reflections present in its TDR response. To accomplish that, both the measured $V_{\text {open }}^{\text {mes }}$ and the direct model of all remaining $N U T_{\text {sim }}^{i}$ are used to reconstruct the wiring network. The design variables are the lengths of different branches $L_{i}$ composing $N U T_{\text {sim }}^{i}$ excluding the first two junction positions $l_{1}$ and $l_{2}$ previously determined.

From the reflectometry data $V_{o p e n}^{\text {mes }}$ of the NUT, the methodology of reconstruction leads to the resolution of an inverse problem: the newton-based algorithm is used minimize the objective function given by equation 7 between $V_{\text {mes }}$ and each $V_{\text {sim }}^{i}$. Accordingly, we will start with an initial population of the $n$ branch lengths $L=\left[L_{1}, L_{2}, L_{n}-2\right]$ composing the $N U T_{\text {sim }}^{i}$. These solutions then undergo the newton-based optimization process described in sec. III-A. After each optimization process the fitness values $F_{v a l}^{i}$ will be sorted and the $N U T_{\text {sim }}^{i}$ with the highest $F_{v a l}^{i}$ will be dropped. This process will be iterated till we reach a stopping criterion demonstrated by the lowest $F_{v a l}^{i}$ corresponding to $N U T_{\text {sim }}^{i}$ whose solution $L$ best fits the branch lengths of the tested network.

$$
F_{v a l}^{i}=\left\|V_{s i m}^{i}-V_{m e s}\right\|_{2}
$$

For clarification purposes, the diagram of Fig. 3 summarizes all the steps we followed in the proposed TDR-GA algorithm.

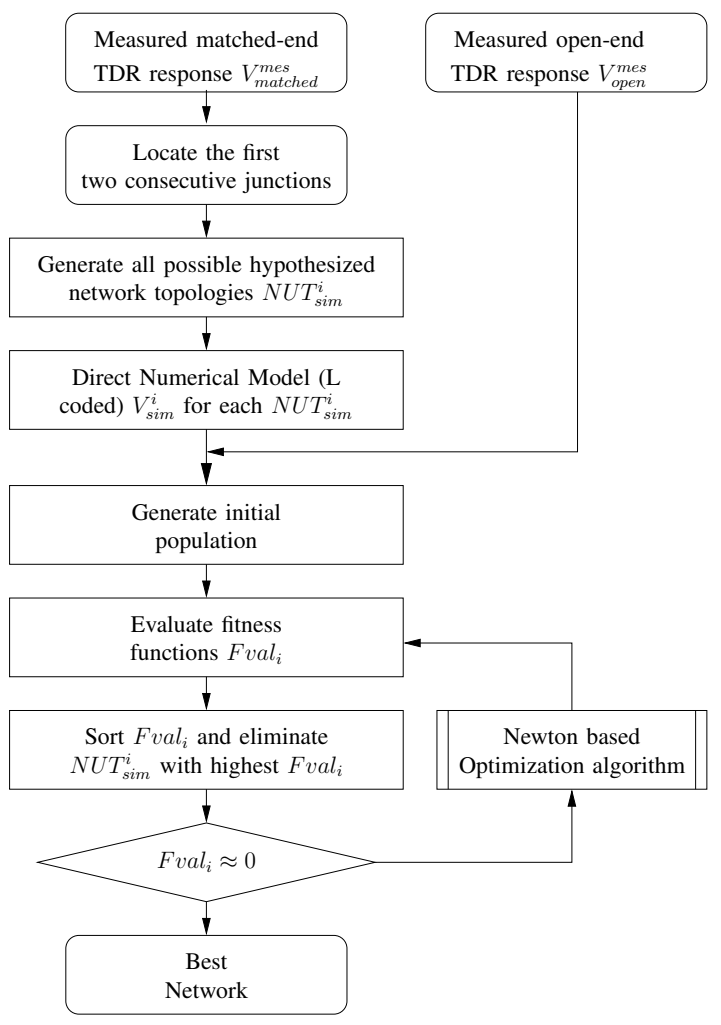

Fig. 3. A diagram illustrating the differebt steps followed by the TDR-GA algorithm.

\section{RESULTS AND DISCUSSIONS}

In order to better illustrate the benefits introduced by the TDR-NEW method in retrieving the topology of an unknown network we opted to test it numerically and experimentally on different NUTs.

\section{A. Numerical Verification}

Numerical results have been deducted using the network configuration already considered in Fig. 1. The same network characteristics and simulation parameters considered in section II-C were followed. The very first step of the TDR-NEW algorithm is to numerically construct all possible hypothesized network topologies based on the diagnosed NUT's number of extremities. To accomplish this task, the analysis of the matched-end reflectometry response $V_{\text {match }}^{\text {mes }}$ of the corresponding NUT as detailed in section II-C shows that the first junction is located at $l_{1}=1.5 \mathrm{~m}$ from the reference testing port $P_{\text {test }}$ and interconnecting $n_{1}=3$ branches. On the other hand, the second consecutive junction appears to be located at $l_{2}=4 \mathrm{~m}$ from $P_{\text {test }}$ while interconnecting $n_{2}=2$ branches. Consequently, based on the presence of $M=6$ extremities and the knowledge of $n_{1}$ and $n_{2}$, we are left by 4 free ports as demonstrated in Fig. 4. Therefore, we can claim that we 

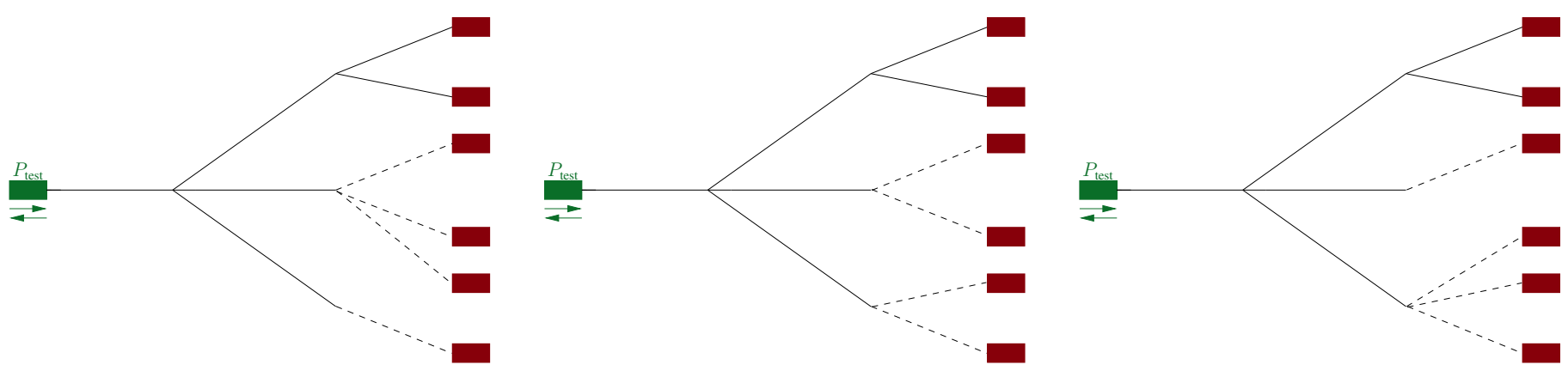

Fig. 4. The hypothesized numerical NUTs constructed based on the 6 extrimities of the tested NUT and the results obtained from the matched-end TDR response analysis.

have 3 possible network configurations $N U T_{\text {sim }}^{i}(i=1,2,3)$ as shown in Figs. 4 among which one resembles the tested network.

To clear up all doubts we will apply the newton-based optimization algorithm to solve the inverse problem between the open-circuited reflectometry response $V_{\text {match }}^{\text {open }}$ and $V_{\text {sim }}^{i}$ of each of the three hypothesized $N U T_{\text {sim }}^{i}$. The number of design length variables of each optimization process depends on the number of branches composing each $N U T_{\text {sim }}^{i}$ excluding the retrieved lengths $l_{1}$ and $l_{2}$. Therefore, continuing the TDR-NEW algorithm detailed in section III-B qualifies the NUT configuration that best resembles the tested NUT as demonstrated in Fig. 5 in a total duration of 123 seconds using a standard state-of-the art computer. The comparison between the obtained NUT with the original tested network shows that the TDR-NEW approach efficiently reconstructed the unknown tested wire network with accurate and precise values of the branch lengths.

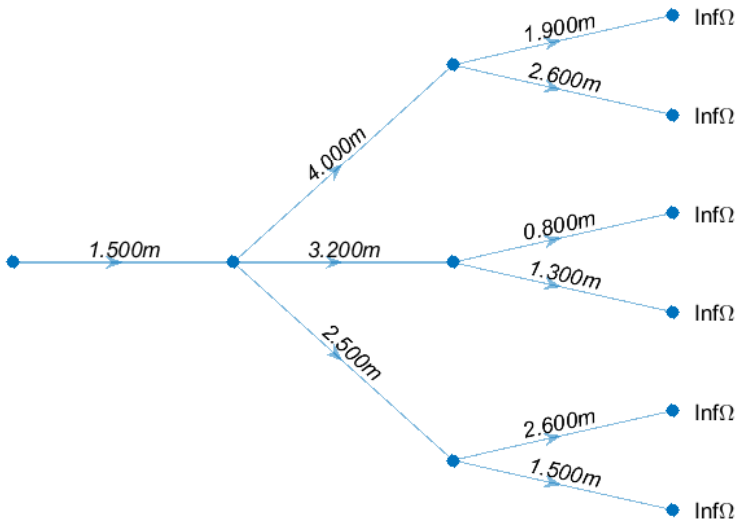

Fig. 5. The constructed NUT after applying the TDR-NEW approach on the numerically simulated TDR response of the NUT of Fig. 1.

\section{B. Experimental Validation}

Experimental tests were also conducted in an effort to validate the proposed TDR-NEW approach on practical reallife networks. We considered a double Y-junction NUT composed of $50 \Omega$ coaxial cables connected as shown in Fig. 6 . The lengths of branches are illustrated on the same figure. A standard TDR measurement was accomplished by injecting a
Gaussian pulse excitation with a $-3 \mathrm{~dB}$ bandwidth of about $2 \mathrm{GHz}$ into the reference testing port of the network. In fact, this process was repeated twice, once while all ports are matched to the characteristic impedance of the cables and another while open-circuiting all extremities. The TDR response is retrieved by measuring the $S_{11}$ parameter of the NUT by a vector network analyzer Rohde \& Schwarz ZVB8 VNA, covering a frequency range from $300 \mathrm{kHz}$ to $8 \mathrm{GHz}$. The reflectograms of Figs. 7 shows the two measured time domain TDR responses of the NUT after applying an inverse fast Fourier transform (IFFT) to the frequency domain measured responses.

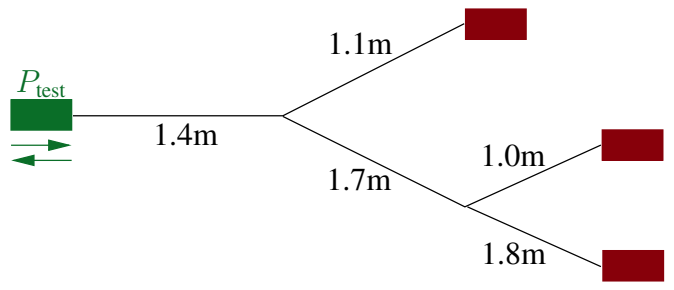

Fig. 6. Layout of the double Y-junction NUT considered for the experimental validation of the TDR-NEW approach including the branch lengths.

The same methodology followed in the numerical verification in section IV-A demonstrating the advantages of the TDR-NEW approach detailed in sec. III-B will be repeated. The resulting reconstructed network is shown in Fig. 8 after a total processing period of 200 seconds; notably it appears to perfectly resemble the tested NUT of Fig. 6. Significantly, a clear close agreement can be noticed from the obtained results.

\section{CONCLUSion}

In this paper we addressed the network reconstruction problem of estimating the topology and the branch lengths of a wire network. The TDR-NEW approach, a non-destructive testing method, was proposed which is based on reflectometry response and newton-based optimization algorithms. The numerical and experimental result has proven the effectiveness and feasibility of the proposed approach in precisely reconstructing unknown branched networks. We showed that the proposed approach is computationally efficient (short processing time), consistent (return correct topology), and robust (can tolerate a maximum level of measurement errors). The framework provides a powerful tool for future implementation on structure and dynamics of large-scale networks. Future 


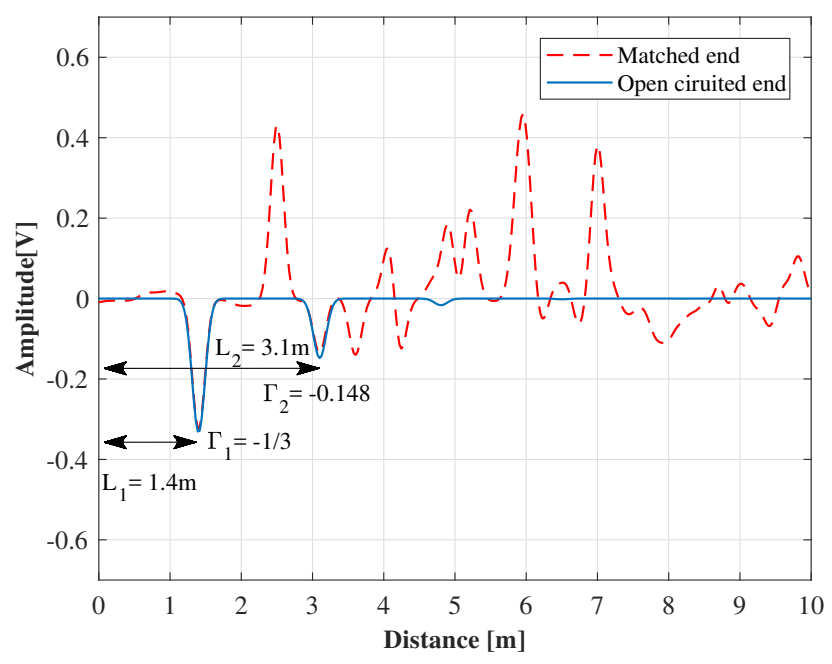

Fig. 7. The TDR reflectometry responses corresponding to the experimental validation of the network of Fig. 6 in the cases of matched and open-ended extremities.

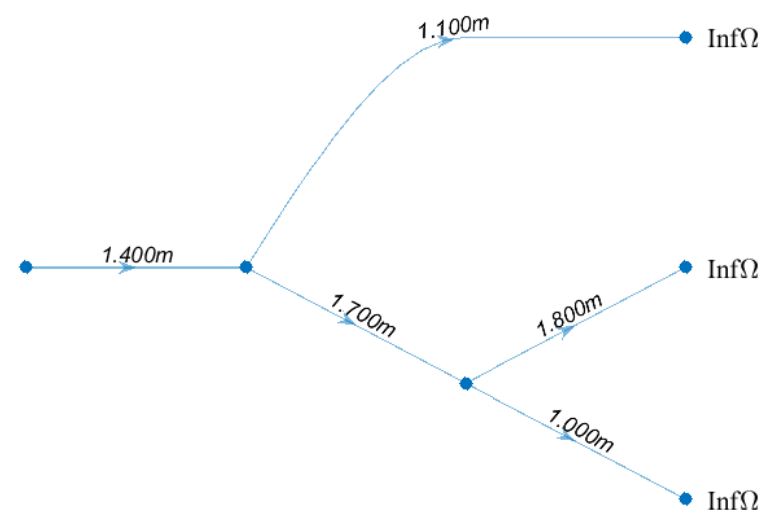

Fig. 8. The constructed NUT after applying the TDR-NEW approach on the measured TDR response of the NUT of Fig. 6.

work will need to deal with advanced global optimization tools, namely the genetic algorithms, for more computational efficiency and shorter processing periods. Besides, it could be interesting to reconstruct the network's topology without having to disconnect the network and force specific measurement frameworks.

\section{REFERENCES}

[1] M. Kafal, "Imaging Techniques for soft fault detection and location in wiring networks," Theses, Université Paris-Saclay, Sep. 2016. [Online].

[2] F. Auzanneau, "Wire troubleshooting and diagnosis: Review and perspectives,"Progress In Electromagnetics Research B, vol. 49, pp. $253-$ 279, 2013.

[3] N. Science and T. C. U. W. S. S. I. W. Group, Review of federal programs for wire system safety: final report. Executive Office of the President of the United States, 2000. [Online].

[4] P. Smith, C. Furse, and J. Gunther, "Analysis of spread spectrum time domain reflectometry for wire fault location," IEEE Sensors Journal, vol. 5, no. 6, pp. 1469-1478, 2005.
[5] L. A. Griffiths, R. Parakh, C. Furse, and B. Baker, "The invisible fray: A critical analysis of the use of reflectometry for fray location," Sensors Journal, IEEE, vol. 6, no. 3, pp. 697-706, 2006.

[6] C. Layer, E. Cabanillas, and J. Benoit, "Using quadrature modulation for precise fault location over wired communication channels," IEEE New Circuits and Systems conference (NEWCAS), pp. 357-360, June 2017.

[7] E. Cabanillas, C. Layer "Automated Phase Offset Correction using Reflectometry in Fault Detection Systems," Proceedins of the 60th IEEE Midwest Symposium on Circuits and Systems (MWSCAS), August 2017.

[8] A. Cozza and L. Pichon, "Echo response of faults in transmission lines: Models and limitations to fault detection," IEEE Transactions on Microwave Theory and Techniques, vol. 64, no. 12, pp. 4155-4164, 2016.

[9] L. Abboud, A. Cozza, and L. Pichon, "A matched-pulse approach for soft-fault detection in complex wire networks," Instrumentation and Measurement, IEEE Transactions on, vol. 61, no. 6, pp. 1719-1732, 2012.

[10] C. Furse, Y. C. Chung, C. Lo, and P. Pendayala, "A critical comparison of reflectometry methods for location of wiring faults," Smart Structures and Systems, vol. 2, no. 1, pp. 25-46, 2006.

[11] M. Kafal and J. Benoit, "Baselining: A critical approach used for soft fault detection in wire networks." EEETEM2017, Beirut, Lebanon, April 2017.

[12] L. Abboud, A. Cozza, and L. Pichon, "A noniterative method for locating soft faults in complex wire networks," Vehicular Technology, IEEE Transactions on, vol. 62, no. 3, pp. 1010-1019, 2013.

[13] M. Kafal, A. Cozza, and L. Pichon, "Locating multiple soft faults in wire networks using an alternative dort implementation," Instrumentation and Measurement, IEEE Transactions on, vol. 65, no. 2, pp. 399-406, 2016.

[14] M. Kafal, A. Cozza, and L. Pichon, "An efficient technique based on dort method to locate multiple soft faults in wiring networks," IEEE Instrumentation \& Measurement Magazine, vol. 19, no. 4, pp. 10-14, 2016.

[15] M. Kafal, A. Cozza, and L. Pichon, "Locating faults with high resolution using single-frequency tr-music processing," IEEE Transactions on Instrumentation and Measurement, vol. 65, no. 10, pp. 2342-2348, 2016.

[16] R. Fletcher, Practical methods of optimization. John Wiley \& Sons, 2013. 